\title{
Synthesis of a New Organic Linker Molecule for Protein Immobilization
}

\author{
Sang Yun Kim and Guncheol Kim \\ Department of Chemistry, College of Natural Science, Chungnam National Wniversity, Daejeon $305-764$, Korea \\ E-mail: guncheoliácnuachr \\ Received Mav 6, 2008
}

Key Words : Linker, Immobilization, Meisenheimer complex, Disulfide

Recent biological and medicinal experimental techniques for diagnosis and drug discovery utilize microarrays of proteins immobilized on solid substrates.' Such protein "chips" can have proteins on surface attached covalently ${ }^{3.3}$ or non-covalently ${ }^{4}$ between proteins and linker molecules frequently by chemisorption of the film components to the substrate from solution. For the well defined systems. organic monolayer films have formed versatile model study. The array's of proteins attached to a solid surface have a great potential for detecting interactions of protein-ligand. proteinprotein. and antibody-antigen.

Among several self-assembling șystems, organosulfur compounds including dialkỵl sulfides. dialkỵl disulfides. and thiols ${ }^{8}$ have been investigated intensively on gold. It has been recognized that self-assembled monolayers (SAM) using organosufur compounds on gold are advantageous over other SAM systems because of higher structural order by densely packing long chains. In addition. versatile functionalization of teminal group at the monolayer surface suggests ideal model șstems for immobilization and interfacial interaction. Several functional groups including anhydride. ${ }^{5}$ succinimidyl ester. ${ }^{113}$ maleimide. ${ }^{13}$ and phosphinothioester ${ }^{12}$ have been applied for the chemoselective binding of proteins.

Here. we report a new potential substrate for the covalent and specific immobilization of nucleophilic molecules. The new organic linker. disulfide 1. has 4, 5-difluoro-2-nitroaniline amide moiety on the head and a long carbon chain with a triazole group. We envisioned that the compound would be prepared wio 1.3-dipolar cycloaddition of azide 2 and alky'ne 3 . $^{13}$ and hoped that a Meisenheimer complex ${ }^{14}$ of 4.5-difluoro-2-nitroaniline amide moiety would serve for a covalent attachment of amine or thiol moieties. and eventually proteins on the surface (Scheme 1).
Prior to the synthesis of the molecule. we wanted to evaluate the reactivity of the substrate 1 toward nucleophilic aromatic substitution. Therefore. we first prepared a anide 4 from 4,5-difluoro-2-nitroaniline and 10-undecenoyl chloride and have tried the substitution reaction with various amines and a thiol as a model study (Table 1). The reaction was carried out in THF/water at room temperature. Primary and secondary amines reacted with the nitroaniline in minutes to afford good yields. Cyclic amines provided better than noncyclic secondary amines and thiol provided the best yield in the shortest reaction time as expected. On the basis of the result. we anticipate the functional groups in proteins. amine or thiol. would show the similar reaction pattem toward the

Table 1. Nucleophilic aromatic substitution reaction in THF/water

\begin{tabular}{|c|c|c|c|c|c|}
\hline & $\mathrm{F}$ & & & 5 & $\mathrm{Nu}$ \\
\hline entry & $\mathrm{NuH}$ & temp & Time $(\mathrm{h})$ & & Yield $(\%)$ \\
\hline 1 & & it & l & $a$ & 98 \\
\hline 2 & & it & 0.5 & b & 97 \\
\hline 3 & & it & 0.25 & c & 96 \\
\hline 4 & & it & 2 & d & 96 \\
\hline 5 & & it & 1 & e & 98 \\
\hline 6 & & it & 0.25 & f & 94 \\
\hline
\end{tabular}<smiles>C=CC(C)(C)n1cc(C(C)C(=O)Nc2cc(F)c(F)cc2[N+](=O)[O-])nn1</smiles>

1<smiles>C#CC(=O)Nc1cc(F)c(F)cc1[N+](=O)[O-]</smiles>

2

Scheme 1 


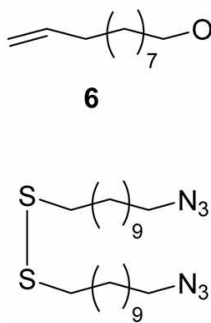

2

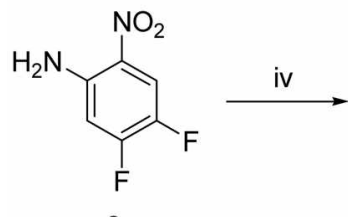

9

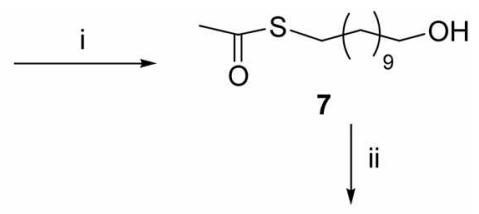

iii

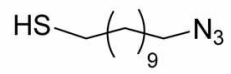

8

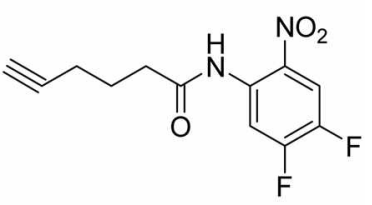

3
Scheme 2. Reagents and conditions: (i) $\mathrm{CH}_{3} \mathrm{COSH}, \mathrm{AIBN}, h$, $56 \%$;ii) a) $\mathrm{MsCl}$, TEA, $\mathrm{CH}_{2} \mathrm{Cl}_{2}$; b) $\mathrm{NaN}_{3}$, HMPA, $40^{\circ} \mathrm{C}, 70 \%$; c) $\mathrm{NaOH}, \mathrm{MeOH} / \mathrm{H}_{2} \mathrm{O}, 47 \%$; (iii) $\mathrm{I}_{2}, \mathrm{O}_{2}, 15 \%$, (iv) 5 -Hexynovl chloride, NaH, THF, $20 \%$.

moiety on gold surface. though with low reactivity.

For the preparation of compound 1. we first treated 10 undecen-1-ol 6 with thioacetic acid in the presence AIBN under U.V. lamp (350 nm) to obtain thioacetate 7 in $56 \%$ yield. ${ }^{15}$ Compound 7 was converted to azathiol 8 via a three step sequence. mesylation (quantitative yield). substitution with $\mathrm{NaN}_{3}(70 \%)$, and hydroly sis $(47 \%)$. Oxidative coupling of aza-thiol 8 using $\mathrm{I}_{2}$ in the presence of $\mathrm{O}_{2}$ afforded azadisulfide 2 in $15 \%$ yield. ${ }^{16}$ Coupling partner compound 3 was readily prepared by treatment of 9 with 5-hexynoyl chloride in $20 \%$ yield (Scheme 2 ).

Coupling reaction to 1 was carried out by Huisgen 1,3dipolar cycloaddition. In particular. the solution of compound 2 and 3 in a 1:1 mixture of water and tert-butyl alcohol in the presence of $\mathrm{CuSO}_{4}$ and sodium ascorbate was stirred under $\mathrm{N}_{2}$ to yield $1 \mathrm{in} 35 \%$ yield. ${ }^{13}$

In conclusion. we sýnthesized a new potential organic linker molecule 1 for protein immobilization on gold surface. Study of formation of self-assembled monolayers of this molecule on gold surface and the reaction with peptides or proteins is under study and the results will be disclosed in due course.

\section{Experimental Section}

A representative procedure for the nucleophilic aromatic substitution reaction of 4

To a solution of $4(80 \mathrm{mg} .0 .24 \mathrm{mmol})$ in $\mathrm{THF} / \mathrm{H}_{2} \mathrm{O}(0.5$ $\mathrm{mL} / 0.5 \mathrm{~mL}$ ) was added propyl anine $(30 \mathrm{mig}, 0.47 \mathrm{~nm}$ ol). The mixture was stirred at room temperature under $\mathrm{N}_{2}$ for $\mathrm{l}$ hr and concentrated. The crude mixture was purified by silica-gel columun chromatography to afford $\mathbf{5 a}(87 \mathrm{mg} .98 \%)$

[4-Fluoro-2-nitro-5-(propyl amino)-phenyl]-10-undecenamide (5a): ${ }^{1} \mathrm{H}-\mathrm{NMR}\left(400 \mathrm{MHz} . \mathrm{CDCl}_{\mathfrak{j}}\right.$ ) $\delta 11.07$ (s. $1 \mathrm{H}), 8.21$ (d. $1 \mathrm{H} . J=8.4 \mathrm{~Hz}) .7 .89(\mathrm{~d}, 1 \mathrm{H}, J=12 \mathrm{~Hz}), 5.75$.
5.85 (m. lH). $4.91-5.00$ (m. $2 \mathrm{H}), 4.84(\mathrm{~s}, \mathrm{lH}) .3 .28$ (q. $2 \mathrm{H} . J$ $=7.2 \mathrm{~Hz}), 2.48(\mathrm{t}, 2 \mathrm{H}, J=7.6 \mathrm{~Hz}), 2.03(\mathrm{q} .2 \mathrm{H}, J=7.2 \mathrm{~Hz}$ ), $1.70-1.77(\mathrm{~m} .4 \mathrm{H}), 1.31-1.4 \mathrm{l}(10 \mathrm{H}), 1.03(\mathrm{t}, 3 \mathrm{H} . J=7.2 \mathrm{~Hz})$.

[t-Fluoro-2-nitro-5-(dimethylamino)-phenyl]-10-undecenamide (5b): ${ }^{1} \mathrm{H}-\mathrm{NMR}\left(400 \mathrm{MHz} . \mathrm{CDCl}_{3}\right) \delta 10.92$ (s, IH). 8.27 (d. IH. $J=8.8 \mathrm{~Hz}), 7.88(\mathrm{~d} .1 \mathrm{H}, J=15.2 \mathrm{~Hz}) .5 .8 \mathrm{l}$ (m, lH). 4.96 (m. $2 \mathrm{H}), 3.16$ (s. $3 \mathrm{H}), 3.15$ (s, 3H), 2.47 (t. $2 \mathrm{H}$, $J=7.6 \mathrm{~Hz}$ ). 2.03 (q. $2 \mathrm{H} . J=7.6 \mathrm{~Hz}$ ). 1.74 (q. $2 \mathrm{H} . J=7.6$ $\mathrm{Hz}), 1.25-1.39(\mathrm{l} 0 \mathrm{H})$.

(t-Fluoro-2-nitro-5-pyrolidinyl-phenyl)-10-undecenamide (5c): ${ }^{1} \mathrm{H}-\mathrm{NMR}\left(400 \mathrm{MHz} . \mathrm{CDCl}_{3}\right) \delta 11.03(\mathrm{~s}, \mathrm{lH})$, $8.11(\mathrm{~d}, \mathrm{IH} . J=8.8 \mathrm{~Hz}) .7 .87(\mathrm{~d}, \mathrm{IH} . J=14.8 \mathrm{~Hz}) .5 .79(\mathrm{~m}$, lH). 4.93 (m, 2H). 3.62 (bs, 4H). 2.46 (t. $2 \mathrm{H}, J=7.6 \mathrm{~Hz}$ ), $2.00(\mathrm{bs}, 6 \mathrm{H}) .1 .74$ (q, 2H, $J=7.6 \mathrm{~Hz}) .1 .26-1.39(\mathrm{l} 0 \mathrm{H})$.

[4-Fluoro-2-nitro-5-(di-n-butyl amino)-phenyl]-10-undecenamide (5d): ${ }^{1} \mathrm{H}-\mathrm{NMR}\left(400 \mathrm{MHz} . \mathrm{CDCl}_{3}\right) \delta 10.95$ (s, IH). 8.29 (d, IH. $J=8.8 \mathrm{~Hz}), 7.86(\mathrm{~d} .1 \mathrm{H}, J=15.6 \mathrm{~Hz}) .5 .80$ $(\mathrm{m}, \mathrm{HH}) .4 .95(\mathrm{~m}, 2 \mathrm{H}), 3.42(\mathrm{t}, 4 \mathrm{H}, J=7.2 \mathrm{~Hz}), 2.45$ (t. $2 \mathrm{H}, J$ $=7.6 \mathrm{~Hz}$ ). 2.03 (q. $2 \mathrm{H}, J=7.2 \mathrm{~Hz}), 1.74$ (q. $2 \mathrm{H}, J=7.6 \mathrm{~Hz}$ ), 1.64 (q. $4 \mathrm{H}, J=7.6 \mathrm{~Hz}), 1.31-1.40(\mathrm{l} 4 \mathrm{H}), 0.96$ (t. $6 \mathrm{H}, J=$ $7.6 \mathrm{~Hz})$.

[4-Fluoro-2-nitro-5-(1-heptane thio)-phenyl]-10-undecenamide (5e): ${ }^{1} \mathrm{H}-\mathrm{NMR}\left(400 \mathrm{MHz}, \mathrm{CDCl}_{3}\right) \delta 10.55$ (s, lH). 8.84 (d, IH. $J=7.2 \mathrm{~Hz}$ ), 7.87 (d. $1 \mathrm{H}, J=10 \mathrm{~Hz}) .5 .79$ (m, lH). 4.99 (m. 2H). 2.96-3.07 (t. 2H. $J=7.2 \mathrm{~Hz}$ ). 2.46$2.70(\mathrm{~m}, 4 \mathrm{H}), 2.04$ (q. $2 \mathrm{H} . J=6.4 \mathrm{~Hz}) .1 .81-1.43(\mathrm{lOH})$, $1.29 \cdot 1.39(10 \mathrm{H}) .0 .88$ (t. $3 \mathrm{H}, J=6.8 \mathrm{~Hz}$ ).

(t-Fluoro-2-nitro-5-piperidinyl-phenyl)-10-undecenamide (5f): ${ }^{1} \mathrm{H}-\mathrm{NMR}\left(400 \mathrm{MHz}, \mathrm{CDCl}_{3}\right) \delta 10.81$ (s. lH), $8.42(\mathrm{~d}, 1 \mathrm{H}, J=8.8 \mathrm{~Hz}) .7 .88(\mathrm{~d}, 1 \mathrm{H} . J=14 \mathrm{~Hz}) .5 .78(\mathrm{~m}$, lH). $4.96(\mathrm{~m}, 2 \mathrm{H}) .3 .38(\mathrm{t}, 4 \mathrm{H} . J=5.2 \mathrm{~Hz}) .2 .47$ (t. $2 \mathrm{H}, J=$ $7.2 \mathrm{~Hz}$ ), 2.03 (q. $2 \mathrm{H} . J=7.2 \mathrm{~Hz}$ ), $1.66-1.78(8 \mathrm{H}) .1 .29-1.41$ $(\mathrm{l} 0 \mathrm{H})$.

11-Hydroxyundecyl ethanethioate (7): To a solution of 10 -undecen-1-ol (500 $\mathrm{mg}, 2.94 \mathrm{mmol}$ ) and AIBN (241 $\mathrm{mg}$. $0.147 \mathrm{mmol}$ ) in $3 \mathrm{~mL}$ of THF was added thioacetic acid (394 mg. 5.87 mmol). The solution was stirred for 14 lur under UV lamp and $2 \mathrm{~mL}$ of water was added to the solution. The mixture was extracted with methylene clloride $(3 \mathrm{~mL} \times 3)$. and the organic layer was dried over $\mathrm{MgSO}_{4}$. Filtration was followed by concentration and purification on silica-gel chromatography to afford $405 \mathrm{mg}$ of 7 in $56 \%$ yield. ${ }^{3} \mathrm{H}$ NMR (400 MHz, $\left.\mathrm{CDCl}_{3}\right) \delta 3.64$ (t. $\left.2 \mathrm{H} . J=6.8 \mathrm{~Hz}\right) .2 .86(\mathrm{t}$, $2 \mathrm{H} . J=7.2 \mathrm{~Hz}), 2.32(\mathrm{~s}, 3 \mathrm{H}) .1 .53-1.60(\mathrm{~m}, 4 \mathrm{H}), 1.27-1.36$ $(\mathrm{l}+\mathrm{H})$ : Mass (EI, m/z) $246\left(\mathrm{M}^{+}\right)$.

11-Azidoundecane-1-thiol (8): A solution of 7, methanesulfonyl chloride (810 mg. $3.29 \mathrm{mmol}$ ). and triethylamine (668 $\mathrm{mg} .6 .58 \mathrm{mmol}$ ) in $3 \mathrm{~mL}$ of methy lene chloride was stirred at room temperature for $2 \mathrm{hr}$. The reaction mixture was quenched with water $(3 \mathrm{~mL})$ and extracted with methlene chloride $(3 \mathrm{~mL} \times 3)$. The organic laver was dried over $\mathrm{MgSO}_{4}$. filtered, and concentrated to afford $1.06 \mathrm{~g}$ of crude mesylate. Without purification. the crude product was treated with sodium azide (690 $\mathrm{mg} .10 .6 \mathrm{mmol}$ ) in HMPA (4 $\mathrm{mL}$ ) at $40^{\circ} \mathrm{C}$ for $2 \mathrm{hr}$. Water $(5 \mathrm{~mL})$ was added to the solution and the mixture was extracted with ether $(3 \mathrm{~mL} \times$ 3). The organic layer was dried over MgSO4. filtered. and 
concentrated. The crude product was purified by colunn chromatography to afford the corresponding azide compound (672 $\mathrm{mg} .70 \%$ ). The azide ( $281 \mathrm{mg} .1 .04 \mathrm{mmol}$ ) was dissolved in $3 \mathrm{~mL}$ of methanol containing $\mathrm{NaOH}$ (166 mig, 4.15 $\mathrm{mm}$ ol). The mixture was stirred at room temperature for 2 lr. The mixture was diluted with methylene chloride $(5 \mathrm{~mL})$ and washed with saturated $\mathrm{NH}_{4} \mathrm{Cl}$. The organic layer was dried over $\mathrm{MgSO}_{4}$, filtered. and concentrated. The crude product was purified by silica-gel colunu chromatography (hexane:ethyl acetate $=20: 1)$ to afford compound $8(112 \mathrm{mg}$. $47 \%$ ). ${ }^{1} \mathrm{H}-\mathrm{NMR}\left(400 \mathrm{MHz} . \mathrm{CDCl}_{3}\right) \delta 3.25$ (t. $2 \mathrm{H} . J=7.2$ $\mathrm{Hz}), 2.52(\mathrm{q}, 2 \mathrm{H}, J=7.6 \mathrm{~Hz}), 1.56-1.62(\mathrm{~m}, 4 \mathrm{H}), 1.28-1.35$ $(\mathrm{l}+\mathrm{H})$; Mass $($ EI. $\mathrm{n} / \mathrm{z}) 229\left(\mathrm{M}^{+}\right)$.

1,2-Bis(11-azidoundecyl)disulfide (2): To a solution of thiol 8 (193 mg, $0.842 \mathrm{mmol})$ in $2 \mathrm{~mL}$ of THF was added I: (64 mg. $0.253 \mathrm{mmol}$ ). $\mathrm{O}_{2}$ was bubbled through the solution for $4 \mathrm{hr}$. The misture was concentrated and purified by silica-gel column chromatography to yield compound 2 (57 mg. $15 \%$ ). ${ }^{1} \mathrm{H}-\mathrm{NMR}\left(400 \mathrm{MHz}, \mathrm{CDCl}_{3}\right) \delta 3.26$ (t. $4 \mathrm{H}, J=$ $6.8 \mathrm{~Hz}), 2.68$ (t. 4 H. $J=7.6 \mathrm{~Hz}) .1 .56-1.69(\mathrm{~m}, 8 \mathrm{H}) .1 .25-$ $1.38(28 \mathrm{H})$; $\mathrm{HRMS} m z\left(\mathrm{M}^{-}\right)$Calcd for $\mathrm{C}_{22} \mathrm{H}_{+4} \mathrm{~N}_{6} \mathrm{~S}_{2}: 456.3069$. Found 456.3065 .

$N$-(4',5'-Difluoro-2'-nitrophenyl)hex-5-ynamide (3): To a solution of 4.5 -difluoro-2-nitroaniline $(1.17 \mathrm{~g} .6 .69 \mathrm{mmol})$ in THF was added $\mathrm{NaH}(161 \mathrm{mg} .6 .69 \mathrm{mmnol})$ at $0{ }^{\circ} \mathrm{C}$. The mixture was stirred at room temperature for $1 \mathrm{hr}$. To the resulting solution was added 5-hexynoyl chloride $(585 \mathrm{mg}$. $4.46 \mathrm{mmol}$ ), and the solution was stirred at room temperature for $t$ hr. Water was added to quench the reaction, and the mixture was extracted with methy lene chloride $(3 \mathrm{~mL} \times$ 3 ). The organic layer was washed with brine, dried over $\mathrm{MgSO}_{4}$, and concentrated. The crude product was purified by silica-gel colunn chromatography to afford conmpound $\mathbf{3}$ (179 mg. 15\%). ${ }^{1} \mathrm{H}-\mathrm{NMR}\left(400 \mathrm{MHz} . \mathrm{CDCl}_{3}\right) \delta 10.50$ (s. lH) $8.84(\mathrm{~m}, \mathrm{lH}) .8 .12(\mathrm{~m} . \mathrm{lH}), 2.67(\mathrm{t}, 2 \mathrm{H}, J=7.2 \mathrm{~Hz})$. 2.35 (dt. $2 \mathrm{H}, J=6.8 \mathrm{~Hz}, J=2.8 \mathrm{~Hz}$ ). 2.03 (t. $\mathrm{lH} . J=2.4 \mathrm{~Hz}$ ). $1.98(\mathrm{~m} .2 \mathrm{H}) ;{ }^{13} \mathrm{C} \cdot \mathrm{NMR}\left(100 \mathrm{MHz} . \mathrm{CDCl}_{3}\right) \delta 172.50,155.51$. $146.30,136.70 .131 .92,119.50,112.10 .83 .70 .71 .25 .35 .40$. 25.50. 20.10: HRMS $m z\left(\mathrm{M}^{-}\right)$Calcd for $\mathrm{C}_{1} \mathrm{H}_{10} \mathrm{~F}_{2} \mathrm{~N}_{3} \mathrm{O}_{3}$ : 268.0659. Found: 268.0650 .

1,2,3-Triazole disulfide (1): To a solution of $2(300 \mathrm{mg}$. $0.658 \mathrm{mmol}$ ) and $3(353 \mathrm{mg} .1 .32 \mathrm{mmol})$ in $4 \mathrm{~mL}$ of tertbutyl alcohol. water. and DMF (1:1:2 mixture) were added sodium ascorbate ( $26 \mathrm{mg} .0 .132 \mathrm{mmol}$ ) and copper sulfate (3 $\mathrm{mg} .0 .013 \mathrm{mmol}$ ). The solution was stirred at room temperature for 15 lu under nitrogen atmosphere. Water was added and the mixture was extracted with methylene chloride (3 $\mathrm{mL} \times 3$ ). The organic layer was dried over $\mathrm{MgSO}_{4}$, filtered. and concentrated. The crude product was purified by silica- gel column chromatography to afford compound 1 ( $220 \mathrm{mg}$. $35 \%$ ). ${ }^{1} \mathrm{H}-\mathrm{NMR}\left(400 \mathrm{MHz}, \mathrm{CDCl}_{3}\right) \delta 10.47$ (s. $\left.2 \mathrm{H}\right) .8 .82(\mathrm{~m}$, lH). 8.09 (m. 2H). 6.97 (d. $2 \mathrm{H} . J=8.4 \mathrm{~Hz}), 4.3 \mathrm{I}$ (t. $4 \mathrm{H} . J=$ $7.2 \mathrm{~Hz}), 2.85$ (t. $4 \mathrm{H} . J=7.6 \mathrm{~Hz}) .2 .67(\mathrm{t} .4 \mathrm{H}, J=7.6 \mathrm{~Hz}$ ), $2.59(\mathrm{t} .4 \mathrm{H}, J=7.6 \mathrm{~Hz}), 2.14(\mathrm{t} .4 \mathrm{H}, J=7.6 \mathrm{~Hz}), 1.23 \cdot 1.32$ $(28 \mathrm{H}) ;{ }^{13} \mathrm{C}-\mathrm{NMR}\left(100 \mathrm{MHz} . \mathrm{CDCl}_{3}\right) \delta 175.2 \mathrm{l}(2 \mathrm{C}), 156.82$ $(2 \mathrm{C}), 145.42(2 \mathrm{C}), 142.11(2 \mathrm{C}), 136.43(2 \mathrm{C}), 132.02(2$ C). $124.45(2 \mathrm{C}) .112 .32(2 \mathrm{C}), 110.21(2 \mathrm{C}), 51.24(2 \mathrm{C})$. 38.79 (2 C). 34.33 (2 C). $31.48(2 \mathrm{C}) \cdot 26.11 \cdot 29.89$ (20 C). Mass (EI. $\mathrm{m} / \mathrm{z}) 496\left(\mathrm{l} / 2 \mathrm{M}^{-}\right)$.

Acknowledgments. This study was financially supported by research fund of Chungnam National University in 2006.

\section{References}

1. (a) Zhu, H.; Snyder. M. Cum Opin. Chem. Biol. 2001. 5 , 40. (b) Cheung. C. L.: Camarero. T. A.: Woods. B. W.: Lin. T. W: Johnsont. I. E.: De Yoreo. I. J. J. Am. Chem. Soc. 2003. 125. 6848. (c) Zhu. H.: Styder. M. Cum Opin. Chent. Biol. 2003. 7. 55. (d) Zhu. H.; Bilgin, M.; Snyder. M. Anmu. Rev Biochem. 2003. 72. 783. (e) Phizicky. E.: Bastiaens, P. I.: Zhu. H.; Snyder. M.; Fields. S. Nature 2003. 122.208

2. (a) Housemant. B. T:: Hulı. J. H.: Kront. S. J.: Mrksich. M. Nat. Biotechnol. 2002. 20. 270. (b) Lesaicherre. M.-L:: Uttamchandani. M.: Chen. G. Y. I.: Yao. S. Q. Bioorg. Med Chem. Lett. 2002. 12. 2079. (c) Toepert. F.: Kriaute. T.: Guffler. S.: Pirés. J. R.: Matzdorf. T.: Oschkinat, H.; Schneider-Mergener, J. Angew. Chem., Iht. Ed. 2003. 42,1136

3. MacBeath. G.: Screiber. S. L. Science 2000, 289. 1760.

4. Zhu. H.: Bilgin. M.: Bangham. R.: Hall. D:: Casamayor. A: Bertone. P.: Lan. N.: Jansen. R.: Bidlingmaier. S.: Houfek. T.: Mitchell. T.: Miller. P.: Dean. R. A.: Gerstein. M.: Styder. M. Science 2001. 293,2101

5. Zisman, W. A. Ad: Chem Ser: 1964, 43. 1

6. Troughton. E. B; Bain. C. D; Whitesides. G. M: Nuzzo, R. G: Allara. D. L.: Porter. M. D. Langmir 1988. 4. 365.

7. Fener. P.: Eberhardt. A.: Eisenberger. P. Science Washington D C.) 1994.266 .1216 and references therein

8. Zhang L.: Lu. T.; Gokel. G. W.: Keifer. A. E. Langmir 1993, 9. 786 and references therein.

9. Chapman. R. G; Ostuni. E.: Yan. L:; Whitesides, G. M. Lamgmir 2000. 16.6927.

10. Fleming. M. S.: Walt. D. R. Lamgnuir 2001. 17. 4836.

11. Hofstee. B. H. J.: Otillio. N. F. Adh: Exp. Med Biol 1974. 42.43.

12. Soellner, M. B.: Dickson, K. A.: Nilsson, B. L.; Raines, R. T. $d$. Am. Chem. Soc. 2003. 125. 11790.

13. Lewis. W. G: Green, L. G: Grynszpan. F; Radic, Z:: Carlier, P. R.: Taylor. P.: Finn. M. G.: Sharpless. K. B. Angew: Chem. Int Ed. 2002. H. 1053.

14. Terrier. F. Chem. Rev: 1982. 82.77

15. Motesharei, K: Myles, D. C. . Am. Chem Soc. 1998, 120. 7328 .

16. Schaeffer, J. R.: Goodhue. C. D; Risle, H. A.: Stevens, R. E. $d$. Org. Chent 1967.32.392. 